\title{
Constraints on an Annihilation Signal from a Core of Constant Dark Matter Density around the Milky Way Center with H.E.S.S.
}

A. Abramowski, ${ }^{1}$ F. Aharonian, ${ }^{2,3,4}$ F. Ait Benkhali, ${ }^{2}$ A. G. Akhperjanian ${ }^{4,5}$ E. O. Angüner, ${ }^{6}$ M. Backes, ${ }^{7}$ S. Balenderan, ${ }^{8}$ A. Balzer, ${ }^{9}$ A. Barnacka, ${ }^{10,11}$ Y. Becherini, ${ }^{12}$ J. Becker Tjus, ${ }^{13}$ D. Berge, ${ }^{14}$ S. Bernhard, ${ }^{15} \mathrm{~K}$. Bernlöhr, ${ }^{2,6}$ E. Birsin, ${ }^{6}$ J. Biteau, ${ }^{16,17}$ M. Böttcher, ${ }^{18}$ C. Boisson, ${ }^{19}$ J. Bolmont, ${ }^{20}$ P. Bordas, ${ }^{21}$ J. Bregeon, ${ }^{22}$ F. Brun, ${ }^{23}$ P. Brun, ${ }^{23}$ M. Bryan, ${ }^{9}$ T. Bulik, ${ }^{24}$ S. Carrigan, ${ }^{2}$ S. Casanova, ${ }^{2,25}$ P. M. Chadwick, ${ }^{8}$ N. Chakraborty, ${ }^{2}$ R. Chalme-Calvet, ${ }^{20}$ R. C. G. Chaves, ${ }^{22}$ M. Chrétien, ${ }^{20}$ S. Colafrancesco, ${ }^{26}$ G. Cologna, ${ }^{27}$ J. Conrad,${ }^{28,29}$ C. Couturier, ${ }^{20}$ Y. Cui, ${ }^{21}$ I. D. Davids, ${ }^{18,7}$ B. Degrange, ${ }^{16}$ C. Deil, ${ }^{2}$ P. deWilt,${ }^{30}$ A. Djannati-Ataï, ${ }^{31}$ W. Domainko, ${ }^{2}$ A. Donath, ${ }^{2}$ L. O'C. Drury, ${ }^{3}$ G. Dubus, ${ }^{32}$ K. Dutson, ${ }^{33}$ J. Dyks, ${ }^{34}$ M. Dyrda ${ }^{25}$ T. Edwards, ${ }^{2}$ K. Egberts,${ }^{35}$ P. Eger, ${ }^{2}$ P. Espigat,${ }^{31}$ C. Farnier, ${ }^{28}$ S. Fegan, ${ }^{16}$ F. Feinstein, ${ }^{22}$ M. V. Fernandes, ${ }^{1}$ D. Fernandez ${ }^{22}$ A. Fiasson, ${ }^{36}$ G. Fontaine, ${ }^{16}$ A. Förster, ${ }^{2}$ M. Füßling, ${ }^{37}$ S. Gabici, ${ }^{31}$ M. Gajdus, ${ }^{6}$ Y. A. Gallant, ${ }^{22}$

T. Garrigoux,${ }^{20}$ G. Giavitto, ${ }^{37}$ B. Giebels, ${ }^{16}$ J. F. Glicenstein, ${ }^{23}$ D. Gottschall, ${ }^{21}$ M.-H. Grondin, ${ }^{22}$ M. Grudzińska, ${ }^{24}$ D. Hadasch, ${ }^{15}$ S. Häffner, ${ }^{38}$ J. Hahn, ${ }^{2}$ J. Harris, ${ }^{3}$ G. Heinzelmann, ${ }^{1}$ G. Henri, ${ }^{32}$ G. Hermann, ${ }^{2}$ O. Hervet, ${ }^{19}$ A. Hillert, ${ }^{2}$ J. A. Hinton, ${ }^{33}$ W. Hofmann, ${ }^{2}$ P. Hofverberg, ${ }^{2}$ M. Holler, ${ }^{35}$ D. Horns, ${ }^{1}$ A. Ivascenko, ${ }^{18}$ A. Jacholkowska, ${ }^{19}$ C. Jahn, ${ }^{38}$ M. Jamrozy, ${ }^{10}$ M. Janiak, ${ }^{34}$ F. Jankowsky, ${ }^{27}$ I. Jung-Richardt, ${ }^{38}$ M. A. Kastendieck, ${ }^{1}$ K. Katarzyński, ${ }^{39}$ U. Katz, ${ }^{38}$ S. Kaufmann ${ }^{27}$ B. Khélifi ${ }^{31}$ M. Kieffer, ${ }^{20}$ S. Klepser, ${ }^{37}$ D. Klochkov, ${ }^{21}$ W. Kluźniak, ${ }^{34}$ D. Kolitzus, ${ }^{15}$ Nu. Komin,${ }^{26}$ K. Kosack, ${ }^{23}$ S. Krakau, ${ }^{13}$ F. Krayzel, ${ }^{36}$ P. P. Krüger, ${ }^{18}$ H. Laffon, ${ }^{40}$ G. Lamanna, ${ }^{36}$ J. Lefaucheur, ${ }^{31}$ V. Lefranc, ${ }^{23}$ A. Lemière, ${ }^{31}$ M. Lemoine-Goumard, ${ }^{40}$ J.-P. Lenain, ${ }^{20}$ T. Lohse, ${ }^{6}$ A. Lopatin, ${ }^{38}$ C.-C. Lu, ${ }^{2}$ V. Marandon, ${ }^{2}$ A. Marcowith, ${ }^{22}$ R. Marx, ${ }^{2}$ G. Maurin,$^{36}$ N. Maxted, ${ }^{22}$ M. Mayer, ${ }^{35}$ T. J. L. McComb,${ }^{8}$ J. Méhault, ${ }^{40}$ P. J. Meintjes, ${ }^{41}$ U. Menzler, ${ }^{13}$ M. Meyer, ${ }^{28}$ A. M. W. Mitchell, ${ }^{2}$ R. Moderski, ${ }^{34}$ M. Mohamed, ${ }^{27}$ K. Morå, ${ }^{28}$ E. Moulin, ${ }^{23}$ T. Murach, ${ }^{6}$ M. de Naurois, ${ }^{16}$ J. Niemiec, ${ }^{25}$ S. J. Nolan, ${ }^{8}$ L. Oakes, ${ }^{6}$ H. Odaka, ${ }^{2}$ S. Ohm, ${ }^{37}$ B. Opitz,${ }^{1}$ M. Ostrowski, ${ }^{10}$ I. Oya, ${ }^{37}$ M. Panter, ${ }^{2}$ R. D. Parsons, ${ }^{2}$ M. Paz Arribas, ${ }^{6}$ N. W. Pekeur, ${ }^{18}$ G. Pelletier, ${ }^{32}$ P.-O. Petrucci, ${ }^{32}$ B. Peyaud, ${ }^{23}$ S. Pita, ${ }^{31}$ H. Poon, ${ }^{2}$ G. Pühlhofer, ${ }^{21}$ M. Punch ${ }^{31}$ A. Quirrenbach, ${ }^{27}$ S. Raab ${ }^{38}$ I. Reichardt, ${ }^{31}$ A. Reimer,${ }^{15}$ O. Reimer, ${ }^{15}$ M. Renaud, ${ }^{22}$ R. de los Reyes, ${ }^{2}$ F. Rieger, ${ }^{2}$ C. Romoli, ${ }^{3}$ S. Rosier-Lees,${ }^{36}$ G. Rowell,${ }^{30}$ B. Rudak,${ }^{34}$ C. B. Rulten, ${ }^{19}$ V. Sahakian,${ }^{5,4}$ D. Salek, ${ }^{42}$ D. A. Sanchez, ${ }^{36}$ A. Santangelo, ${ }^{21}$ R. Schlickeiser, ${ }^{13}$ F. Schüssler, ${ }^{23}$ A. Schulz, ${ }^{37}$ U. Schwanke, ${ }^{6}$ S. Schwarzburg, ${ }^{21}$ S. Schwemmer, ${ }^{27}$ H. Sol ${ }^{19}$ F. Spanier, ${ }^{18}$ G. Spengler, ${ }^{28}$ F. Spies, ${ }^{1}$ Ł. Stawarz,${ }^{10}$ R. Steenkamp, ${ }^{7}$ C. Stegmann, $, 35,37$ F. Stinzing ${ }^{38}$ K. Stycz ${ }^{37}$ I. Sushch, ${ }^{6,18}$ J.-P. Tavernet, ${ }^{20}$ T. Tavernier, ${ }^{31}$ A. M. Taylor, ${ }^{3}$ R. Terrier, ${ }^{31}$ M. Tluczykont, ${ }^{1}$ C. Trichard,${ }^{36}$ K. Valerius, ${ }^{38}$ C. van Eldik,${ }^{38}$ B. van Soelen, ${ }^{41}$ G. Vasileiadis, ${ }^{22}$ J. Veh, ${ }^{38}$ C. Venter, ${ }^{18}$ A. Viana, ${ }^{2}$ P. Vincent,${ }^{20}$ J. Vink, ${ }^{9}$ H. J. Völk, ${ }^{2}$ F. Volpe, ${ }^{2}$ M. Vorster, ${ }^{18}$ T. Vuillaume, ${ }^{32}$ S. J. Wagner, ${ }^{27}$ P. Wagner, ${ }^{6}$ R. M. Wagner, ${ }^{28}$ M. Ward, ${ }^{8}$ M. Weidinger, ${ }^{13}$ Q. Weitzel, ${ }^{2}$ R. White, ${ }^{33}$ A. Wierzcholska, ${ }^{25}$ P. Willmann, ${ }^{38}$ A. Wörnlein, ${ }^{38}$ D. Wouters, ${ }^{23}$ R. Yang, ${ }^{2}$ V. Zabalza, ${ }^{2,33}$ D. Zaborov, ${ }^{16}$ M. Zacharias, ${ }^{27}$ A. A. Zdziarski, ${ }^{34}$ A. Zech, ${ }^{19}$ and H.-S. Zechlin ${ }^{1}$

\section{(H.E.S.S. Collaboration)}

\section{${ }^{1}$ Universität Hamburg, Institut für Experimentalphysik, Luruper Chaussee 149, D 22761 Hamburg, Germany \\ ${ }^{2}$ Max-Planck-Institut für Kernphysik, P.O. Box 103980, D 69029 Heidelberg, Germany ${ }^{3}$ Dublin Institute for Advanced Studies, 31 Fitzwilliam Place, Dublin 2, Ireland}

${ }^{4}$ National Academy of Sciences of the Republic of Armenia, Marshall Baghramian Avenue, 24, O019 Yerevan, Republic of Armenia

${ }^{5}$ Yerevan Physics Institute, 2 Alikhanian Brothers St., 375036 Yerevan, Armenia

${ }^{6}$ Institut für Physik, Humboldt-Universität zu Berlin, Newtonstr. 15, D 12489 Berlin, Germany

${ }^{7}$ University of Namibia, Department of Physics, Private Bag 13301, Windhoek, Namibia

${ }^{8}$ University of Durham, Department of Physics, South Road, Durham DH1 3LE, United Kingdom

${ }^{9}$ GRAPPA, Anton Pannekoek Institute for Astronomy, University of Amsterdam, Science Park 904, 1098 XH Amsterdam, Netherlands

${ }^{10}$ Obserwatorium Astronomiczne, Uniwersytet Jagielloński, ul. Orla 171, 30-244 Kraków, Poland

${ }^{11}$ Now at Harvard-Smithsonian Center for Astrophysics, 60 Garden St, MS-20, Cambridge, Massachusetts 02138, USA

${ }^{12}$ Department of Physics and Electrical Engineering, Linnaeus University, 35195 Växjö, Sweden

${ }^{13}$ Institut für Theoretische Physik, Lehrstuhl IV: Weltraum und Astrophysik, Ruhr-Universität Bochum, D 44780 Bochum, Germany

${ }^{14}$ GRAPPA, Anton Pannekoek Institute for Astronomy and Institute of High-Energy Physics, University of Amsterdam, Science Park 904, 1098 XH Amsterdam, Netherlands

${ }^{15}$ Institut für Astro- und Teilchenphysik, Leopold-Franzens-Universität Innsbruck, A-6020 Innsbruck, Austria

${ }^{16}$ Laboratoire Leprince-Ringuet, Ecole Polytechnique, CNRS/IN2P3, F-91128 Palaiseau, France

${ }^{17}$ Now at Santa Cruz. Institute for Particle Physics, Department of Physics, University of California at Santa Cruz, Santa Cruz, California 95064, USA 


\author{
${ }^{18}$ Centre for Space Research, North-West University, Potchefstroom 2520, South Africa \\ ${ }^{19}$ LUTH, Observatoire de Paris, CNRS, Université Paris Diderot, 5 Place Jules Janssen, 92190 Meudon, France \\ ${ }^{20}$ LPNHE, Université Pierre et Marie Curie Paris 6, Université Denis Diderot Paris 7, CNRS/IN2P3, \\ 4 Place Jussieu, F-75252 Paris Cedex 5, France \\ ${ }^{21}$ Institut für Astronomie und Astrophysik, Universität Tübingen, Sand 1, D 72076 Tübingen, Germany \\ ${ }^{22}$ Laboratoire Univers et Particules de Montpellier, Université Montpellier 2, CNRS/IN2P3, CC 72, Place Eugène Bataillon, \\ F-34095 Montpellier Cedex 5, France \\ ${ }^{23}$ DSM/Irfu, CEA Saclay, F-91191 Gif-Sur-Yvette Cedex, France \\ ${ }^{24}$ Astronomical Observatory, The University of Warsaw, Al. Ujazdowskie 4, 00-478 Warsaw, Poland \\ ${ }^{25}$ Instytut Fizyki Jadrowej PAN, ul. Radzikowskiego 152, 31-342 Kraków, Poland \\ ${ }^{26}$ School of Physics, University of the Witwatersrand, 1 Jan Smuts Avenue, Braamfontein, Johannesburg 2050, South Africa \\ ${ }^{27}$ Landessternwarte, Universität Heidelberg, Königstuhl, D 69117 Heidelberg, Germany \\ ${ }^{28}$ Oskar Klein Centre, Department of Physics, Stockholm University, Albanova University Center, SE-10691 Stockholm, Sweden \\ ${ }^{29}$ Wallenberg Academy Fellow \\ ${ }^{30}$ School of Chemistry \& Physics, University of Adelaide, Adelaide 5005, Australia \\ ${ }^{31}$ APC, AstroParticule et Cosmologie, Université Paris Diderot, CNRS/IN2P3, CEA/Irfu, Observatoire de Paris, Sorbonne Paris Cité, \\ 10, rue Alice Domon et Léonie Duquet, 75205 Paris Cedex 13, France \\ ${ }^{32}$ Univ. Grenoble Alpes, IPAG, F-38000 Grenoble, France and CNRS, IPAG, F-38000 Grenoble, France \\ ${ }^{33}$ Department of Physics and Astronomy, The University of Leicester, University Road, Leicester LE1 7RH, United Kingdom \\ ${ }^{34}$ Nicolaus Copernicus Astronomical Center, ul. Bartycka 18, 00-716 Warsaw, Poland \\ ${ }^{35}$ Institut für Physik und Astronomie, Universität Potsdam, Karl-Liebknecht-Strasse 24/25, D 14476 Potsdam, Germany \\ ${ }^{36}$ Laboratoire d'Annecy-le-Vieux de Physique des Particules, Université de Savoie, CNRS/IN2P3, F-74941 Annecy-le-Vieux, France \\ ${ }^{37}$ DESY, D-15738 Zeuthen, Germany \\ ${ }^{38}$ Universität Erlangen-Nürnberg, Physikalisches Institut, Erwin-Rommel-Str. 1, D 91058 Erlangen, Germany \\ ${ }^{39}$ Centre for Astronomy, Faculty of Physics, Astronomy and Informatics, Nicolaus Copernicus University, \\ Grudziadzka 5, 87-100 Torun, Poland \\ ${ }^{40}$ Université Bordeaux 1, CNRS/IN2P3, Centre d'Études Nucléaires de Bordeaux Gradignan, 33175 Gradignan, France \\ ${ }^{41}$ Department of Physics, University of the Free State, PO Box 339, Bloemfontein 9300, South Africa \\ ${ }^{42}$ GRAPPA, Institute of High-Energy Physics, University of Amsterdam, Science Park 904, 1098 XH Amsterdam, Netherlands
}

(Received 12 October 2014; published 24 February 2015)

An annihilation signal of dark matter is searched for from the central region of the Milky Way. Data acquired in dedicated on-off observations of the Galactic center region with H.E.S.S. are analyzed for this purpose. No significant signal is found in a total of $\sim 9 \mathrm{~h}$ of on-off observations. Upper limits on the velocity averaged cross section, $\langle\sigma v\rangle$, for the annihilation of dark matter particles with masses in the range of $\sim 300 \mathrm{GeV}$ to $\sim 10 \mathrm{TeV}$ are derived. In contrast to previous constraints derived from observations of the Galactic center region, the constraints that are derived here apply also under the assumption of a central core of constant dark matter density around the center of the Galaxy. Values of $\langle\sigma v\rangle$ that are larger than $3 \times 10^{-24} \mathrm{~cm}^{3} / \mathrm{s}$ are excluded for dark matter particles with masses between $\sim 1$ and $\sim 4 \mathrm{TeV}$ at $95 \%$ C.L. if the radius of the central dark matter density core does not exceed $500 \mathrm{pc}$. This is the strongest constraint that is derived on $\langle\sigma v\rangle$ for annihilating $\mathrm{TeV}$ mass dark matter without the assumption of a centrally cusped dark matter density distribution in the search region.

DOI: 10.1103/PhysRevLett.114.081301

Introduction.-The formation of the large scale structure of the universe as well as the dynamics of galaxy clusters and individual galaxies strongly suggest the presence of dark matter on the respective length scale [1]. Many extensions of the standard model of particle physics predict a stable particle without electromagnetic coupling whose presence can account for the missing mass that is apparent in astrophysical environments [1]. The annihilation of dark matter particles is expected to produce photons with energies up to the mass of the dark matter particles [2]. The detection of $\gamma$ rays from a given direction can thus indirectly probe the presence of dark matter particles along the corresponding line of sight.
PACS numbers: 95.35.+d, 14.80.Ly, 95.85.Pw, 98.35.Gi

The central region of the Milky Way is of particular interest for indirect searches for annihilating dark matter because the squared dark matter density integrated over the line of sight towards the target region (i.e., the astrophysical or $J$ factor) is expected to be large [3]. The $J$ factor for observations of the Galactic center region depends strongly on the dark matter density distribution within the Milky Way. Simulations of the dynamics of the dark matter content of galaxies predict to universal dark matter density distributions. Towards the center of the galaxies, the influence of baryons on the distribution of dark matter is not yet resolved. The formation of pronounced density cusps towards the center of galaxies $([4,5])$ and, more 


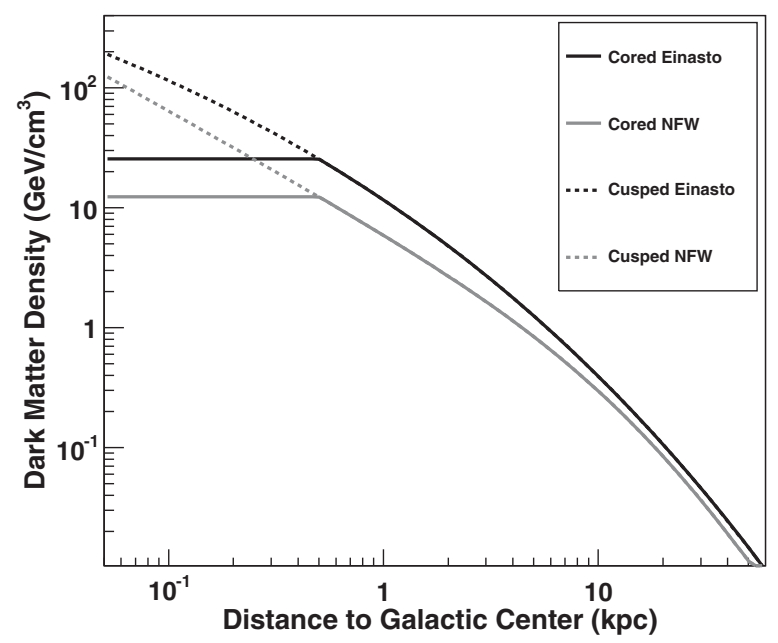

FIG. 1. Dark matter density as a function of the distance to the Galactic center. The parametrizations for the cusped Einasto and NFW profiles are taken from [10]. The cored Einasto and NFW density profiles follow the respective cusped profiles at distances to the Galactic center that are larger than the core radius of $500 \mathrm{pc}$.

recently, the prediction that the dark matter density in the central few hundred pc is almost constant [6-8] have been discussed. The latter prediction of an almost constant dark matter density in the central region of the Galaxy is considered in this Letter.

The current strongest constraints on the velocity averaged cross section for the self-annihilation of dark matter particles with masses in the range of $\sim 400 \mathrm{GeV}$ to $\sim 10 \mathrm{TeV}$ result from a search for an extended emission of $\gamma$ rays in the central region of the Milky Way with H.E.S.S. [9]. However, the constraints apply only if the dark matter density distribution in the central $500 \mathrm{pc}$ of the Milky Way is cusped (see dotted lines in Fig. 1). An alternative search for the annihilation of dark matter particles is presented in this Letter and strong constraints on $\langle\sigma v\rangle$ are derived without the assumption of a dark matter density profile that is cusped in the central $500 \mathrm{pc}$ of the Milky Way.

The high energy stereoscopic system.-The high energy stereoscopic system (H.E.S.S.) is an array of imaging atmospheric Cherenkov telescopes (IACT) in the Namibian Khomas Highland. IACTs detect the Cherenkov light emitted by electromagnetic showers that are induced when primary $\gamma$ rays interact with air nuclei in Earth's atmosphere. Charged cosmic rays also induce showers in Earth's atmosphere and constitute background for the IACT detection of $\gamma$ rays. Cosmic ray background events that cannot be suppressed during the analysis of H.E.S.S. data (see [11]) are typically treated with a background subtraction technique [12]. The background subtraction relies on the definition of a signal region for which a background region is constructed. The construction of the background region must be performed such that the ratio of the acceptance for background events in the signal and the background region is known from instrumental characteristics. The definition of

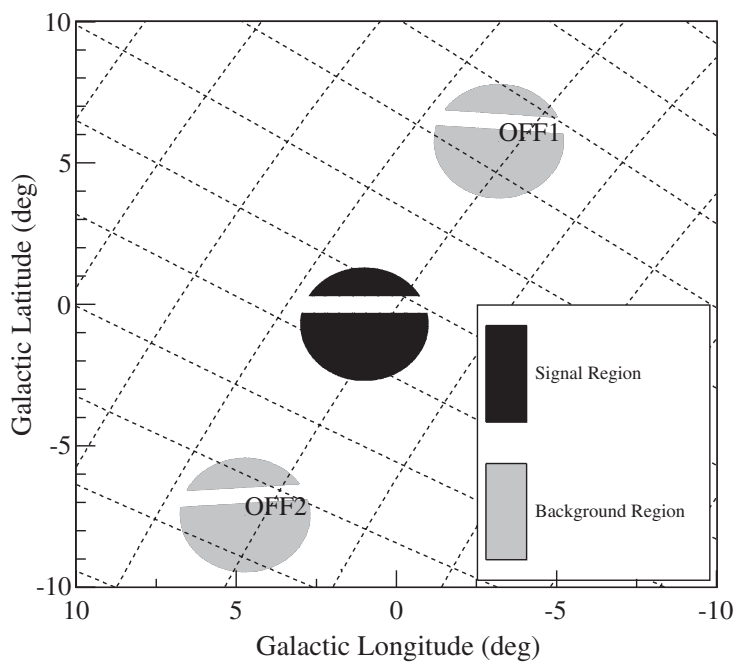

FIG. 2. The signal region close to the Galactic center and the two background regions with a symmetric right ascension offset of \pm 35 min to the signal region. A right ascension-declination coordinate grid is overlaid. The exclusion of the Galactic plane $\left(|b|<0.3^{\circ}\right)$ in the signal region and the regions with a $\pm 35 \mathrm{~min}$ offset in right ascension to $|b|<0.3^{\circ}$ in the background regions are visible.

the background region enables a comparison between the number of events that are detected in the signal region and the number of background events that are expected in the signal region. The acceptance for background events of H.E.S.S. is in general strongly influenced by atmospheric conditions, the pointing zenith angle and the night sky background in the observed field of view. See [11] for more information on the H.E.S.S. experiment.

On-off observations of the Galactic center region with H.E.S.S.-The on-off observation mode (see also [13], [12]) refers in this Letter to a special observation strategy where a background (off1) region, the signal (on) region, and another background (off2) region are observed consecutively for $33 \mathrm{~min}$ each. Figure 2 shows the observed regions in galactic coordinates. The signal region has a radius of $2^{\circ}$ and centers at $l=1^{\circ}, b=-0.7^{\circ}$ in galactic coordinates or $\alpha=267.7^{\circ}, \delta=-28.4^{\circ}$ in equatorial coordinates (J2000). The centers of the two background regions have a symmetric offset of $\pm 35 \mathrm{~min}$ in right ascension to the signal region center. The two minute difference between the right ascension offset between the signal and background regions and the observation length allows for a transition time between the observations. The on-off observation pattern allows the equalization of the azimuth and zenith angles that are covered by array pointings in each of the observations. Differences in the acceptance for background events which result from differences in the zenith angle array pointing range can thus be neglected. The time difference of $35 \mathrm{~min}$ between the observations is a compromise between the demand for small atmospheric changes (i.e., small time differences) and a large offset in 


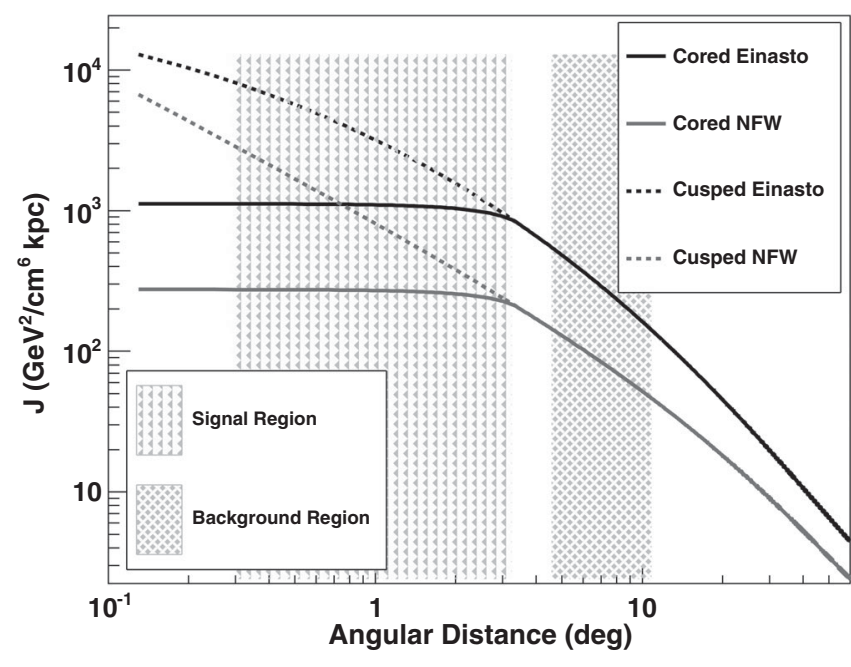

FIG. 3. Line of sight integral over the squared dark matter density as a function of the angular distance between the direction of the line of sight and the direction of the Galactic center. A $500 \mathrm{pc}$ core radius is assumed for the cored dark matter density profiles. Overlaid is the range of angular distances to the Galactic center covered by the signal and background regions of the off1-on-off2 data sets.

right ascension (i.e., large time differences). Two background regions are observed, to better control residual imbalances in the acceptance for background events between the observations. Figure 3 shows the $J$ factor for a given line of sight as a function of the angular distance, $\theta$, between the directions of the line of sight and the Galactic center. The $J$ factor is proportional to the expected number of dark matter annihilation events in the respective direction. The $\theta$ angle ranges that are covered by the signal and background regions in the off1-on-off2 observations are indicated in Fig. 3. It is concluded from this figure that the expected number of dark matter annihilation events is larger in the signal than in the background regions when the radius of the core of constant dark matter density around the Galactic center is $500 \mathrm{pc}$ or less. This is a clear advantage of the on-off method when compared to the background subtraction technique that is applied in [9] which relies on the simultaneous observation of the Galactic center region and a background region in the same finite H.E.S.S. field of view with $\sim 2^{\circ}$ radius.

The application of standard quality criteria for H.E.S.S. data [11] and the additional requirement for compatible instrumental and atmospheric conditions within an off1-onoff 2 observation result in a total of six off1-on-off2 data sets. All data sets were taken within one week in 2010 with the H.E.S.S. I array of four identical IACTs. The total dead time corrected observation time for each of the three observed regions is $3.05 \mathrm{~h}$. The mean zenith angle of the array pointing for the data sets is $12^{\circ}$.

Data analysis.-The image cleaning (see [11]) low and high pixel intensity thresholds for the data are chosen to be 7 pe (photo electrons) and 10 pe. Using the observed distribution of pixel intensities in cosmic ray events, it was checked that these image cleaning cut criteria eliminate effects due to differences in sky brightness between the observed regions. Standard Hillas criteria [11] for the selection of $\gamma$-ray events are applied to the data. The thresholds used for image cleaning lead to an energy threshold of $290 \mathrm{GeV}$. Only events with reconstructed directions within the central $2^{\circ}$ angular distance around the pointing position of each observation are considered to account for the truncation of $\gamma$-ray images near the edges of the H.E.S.S. field of view. The Galactic plane $\left(|b|<0.3^{\circ}\right)$ is excluded from the analysis to avoid the detection of $\gamma$ rays from astrophysical sources (e.g., the Galactic center source HESSJ1745-290, [14]) without relation to dark matter annihilation. The exclusion region is shifted by the respective pointing position offset in right ascension into the two background regions to equalize the acceptance in the signal and background regions (see Fig. 2). To rule out the detection of $\gamma$ rays from astrophysical sources, the considered data with the chosen exclusion regions are analyzed with the ring background [12] method and a correlation radius of 0.1 deg prior to the on-off analysis. The resulting skymaps of the three observed regions show no indication for a significant excess. It is concluded from the analysis with the ring background method that the chosen exclusion regions are sufficient to exclude astrophysical sources of gamma rays for the on-off analysis.

The mean exposure ratio, $\alpha=0.5$, for the on-off data analysis is the ratio of the live times for the observation of the signal and background regions [12]. However, imbalances in the acceptance for background events between the signal region and the two background regions lead to a systematic error, $\sigma_{\alpha}$, on the exposure ratio. A conservative estimate for the relative systematic error on the exposure ratio, $\sigma_{\alpha} / \alpha=2 \%$, is derived. This estimate results from a comparison of the number of events which pass $\gamma$-ray event selection criteria in the two background regions.

Results. - A total of $N_{\text {on }}=24268$ signal and $N_{\text {off }}=$ 49028 background events are measured that pass standard Hillas criteria [11] for the selection of $\gamma$-ray events. The total $\gamma$-ray signal $s$ has a statistical significance of $-0.5 \sigma$. The statistical significance is calculated with the log-likelihood ratio test statistic as described in [15] with the likelihood function (see also [16])

$$
L=P\left(N_{\mathrm{on}}, \hat{\alpha} b+s\right) P\left(N_{\mathrm{off}}, b\right) G\left(\hat{\alpha}, \alpha, \sigma_{\alpha}\right) .
$$

Here, $P$ and $G$ represent the Poisson and Gaussian distributions. The parameters $b$ (mean number of background events) and $\hat{\alpha}$ (exposure ratio with mean $\alpha$ ) are treated as nuisance parameters. For comparison, the significance of the $\gamma$-ray event excess as calculated with Eq. (17) in [17] without consideration of the systematic error on the exposure ratio is $-1.3 \sigma$. Since no significant $\gamma$-ray signal is measured, an upper limit on the integrated $\gamma$-ray signal for energies ranging from the instrumental 


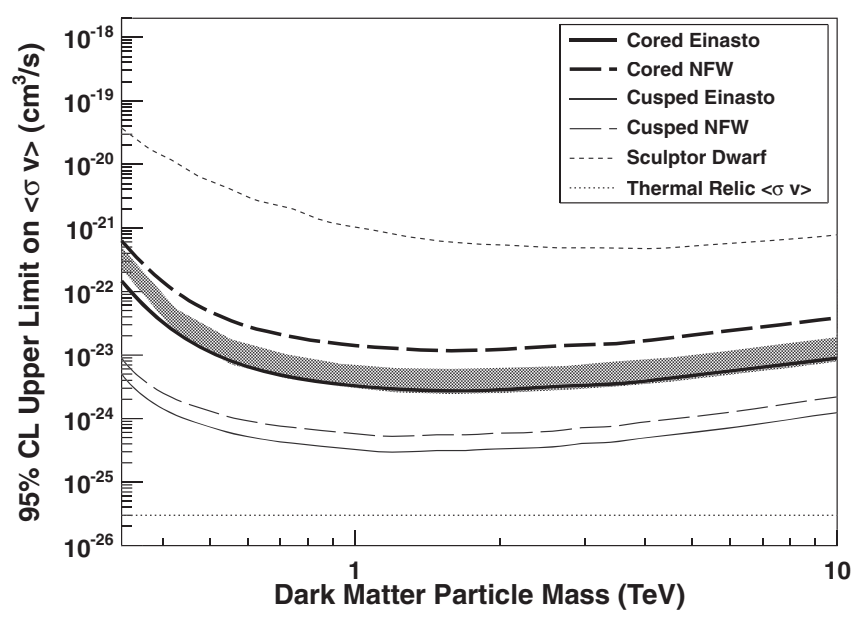

FIG. 4. Upper limits on the velocity averaged dark matter self annihilation cross section as a function of the dark matter particle mass. The upper limits for the cored Einasto and NFW density profiles hold for a core radius of $500 \mathrm{pc}$ and the annihilation of dark matter particles into light quarks ([2]). The filled area around the upper limit curve for the cored Einasto dark matter profile shows the $\pm 1 \sigma$ variations around the upper limit that is expected for this dark matter density profile when no annihilation signal is detected. The derived upper limit is stronger than the expected upper limit due to the negative significance of the measured excess. For comparison, the velocity averaged annihilation cross section of a thermal relic dark matter particle is shown. Additionally shown are the upper limits that are derived in [9] for cusped Einasto and NFW profiles as well as the upper limit that is derived in [19] for a cored dark matter density distribution around the Sculptor dwarf galaxy.

energy threshold to a maximum energy $\hat{E}$ is derived. For the calculation of the upper limit, the likelihood function that is given by Eq. (1) is analyzed with the method described in [15]. The upper limit on the energy integrated signal translates (see, e.g., [18]) into an upper limit on the velocity averaged dark matter self annihilation cross section, $\langle\sigma v\rangle(M)$, for a dark matter particle with mass $M=\hat{E}$. The variation of the instrumental response with the zenith and azimuth angles of the array pointing and within the field of view is accounted for in the analysis. The consideration of the $2 \%$ relative systematic error on the exposure ratio increases the upper limit on $\langle\sigma v\rangle$ by a factor of $\sim 3$. Upper limits on $\langle\sigma v\rangle$ are presented in Fig. 4 for Einasto and NFW dark matter density profiles with a 500 pc radius core of constant dark matter density around the Galactic center. The parameters for the NFW and Einasto density profiles are taken from [10]. The derived upper limits on $\langle\sigma v\rangle$ hold for the $\gamma$-ray energy spectrum that is expected from the self annihilation of dark matter particles into light quarks (see [2], the same spectrum is assumed in [9]). For an Einasto dark matter profile that is cored in the inner $500 \mathrm{pc}$ around the Galactic center, values of $\langle\sigma v\rangle \sim 3 \times 10^{-24} \mathrm{~cm}^{3} / \mathrm{s}$ or larger are excluded for dark matter particle masses in between $\sim 1$ to $\sim 4 \mathrm{TeV}$ at $95 \%$ C.L. The upper limits on
TABLE I. Field of view averaged astrophysical factors for the signal (subscript on) and for the live time weighted average of the two background regions (subscript off). The values are in units of $\mathrm{GeV}^{2} \mathrm{~cm}^{-6} \mathrm{kpc}$ and are tabled for Einasto and NFW profiles as a function of the radius $(R)$ of the central dark matter core.

\begin{tabular}{lcccc}
\hline \hline$R(\mathrm{kpc})$ & $J_{\text {on }}^{\text {Einasto }}$ & $J_{\text {off }}^{\text {Einasto }}$ & $J_{\text {on }}^{\mathrm{NFW}}$ & $J_{\text {off }}^{\mathrm{NFW}}$ \\
\hline 0 & 2167 & 268 & 559 & 78 \\
0.5 & 1036 & 268 & 256 & 78 \\
0.75 & 636 & 268 & 165 & 78 \\
1 & 426 & 255 & 117 & 75 \\
2 & 138 & 126 & 46 & 43 \\
\hline \hline
\end{tabular}

$\langle\sigma v\rangle$ that are derived for an Einasto dark matter density distribution with a core radius of $500 \mathrm{pc}$ are the most constraining exclusions that are derived for $\mathrm{TeV}$ mass dark matter without the assumption of a centrally cusped dark matter density distribution in the search region. However, these limits are one order of magnitude less constraining than the current best limits for cusped dark matter density distributions (see Fig. 4) and 2 orders of magnitudes weaker than the expectation for thermal relic dark matter (see, e.g., [1]).

For core radii different from $500 \mathrm{pc}$, the upper limit on the velocity averaged dark matter self annihilation cross section scales like $\langle\sigma v\rangle_{\mathrm{R}}=\left(\Delta J_{500 \mathrm{pc}} / \Delta J_{\mathrm{R}}\right)\langle\sigma v\rangle_{500 \mathrm{pc}}$ where $\Delta J$ denotes the difference between the field of view averaged astrophysical factors in the signal and background region and the subscript is equal to the core radius. The field of view averaged astrophysical factors in the signal and background region of the considered on-off analysis for different core radii are listed in Table I. The upper limits on $\langle\sigma v\rangle$ increase by a factor of 2 (5) if the radius of the central core of constant dark matter density is $750 \mathrm{pc}(1 \mathrm{kpc})$ when compared to a core radius of $500 \mathrm{pc}$.

Summary.-A search for a signal from annihilating dark matter around the Galactic center was performed. For this purpose, data that were acquired in dedicated on-off observations of the Galactic center region with H.E.S.S. were analyzed. No significant signal was found. The employed observation technique enabled the derivation of upper limits on $\langle\sigma v\rangle$ that are significantly more conservative in respect to the distribution of dark matter in the Galactic center region than previous constraints. In particular, the constraints apply also under the assumption of a core of constant dark matter density around the Galactic center. If the dark matter density in the central $500 \mathrm{pc}$ around the Galactic center is constant and follows outside of the core radius an Einasto profile, values of $\langle\sigma v\rangle$ that are larger than $3 \times 10^{-24} \mathrm{~cm}^{3} / \mathrm{s}$ were excluded for dark matter particle masses between $\sim 1$ and $\sim 4 \mathrm{TeV}$ at $95 \%$ C.L. This is currently the best constraint on $\langle\sigma v\rangle$ that has been derived without the assumption of a centrally cusped dark matter density distribution in the search region. 
The support of the Namibian authorities and of the University of Namibia in facilitating the construction and operation of H.E.S.S. is gratefully acknowledged, as is the support of the German Ministry of Education and Research (BMBF), the Max Planck Society, the French Ministry of Research, the CNRS-IN2P3, and the Astroparticle Interdisciplinary Programme of the CNRS, the United Kingdom Particle Physics and Astronomy Research Council (PPARC), the IPNP of the Charles University, the South African Department of Science and Technology and National Research Foundation, and by the University of Namibia. We appreciate the excellent work of the technical support staff in Berlin, Durham, Hamburg, Heidelberg, Palaiseau, Paris, Saclay, and in Namibia in the construction and operation of the equipment. J. Méhault is funded by contract ERC-StG-259391 from the European Community

[1] G. Bertone, D. Hooper, and J. Silk, Phys. Rep. 405, 279 (2005).

[2] A. Tasitsiomi and A. V. Olinto, Phys. Rev. D 66, 083006 (2002).
[3] L. Bergstrom, P. Ullio, and J. H. Buckley, Astropart. Phys. 9, 137 (1998).

[4] O. Y. Gnedin and J. R. Primack, Phys. Rev. Lett. 93, 061302 (2004).

[5] L. Sadeghian, F. Ferrer, and C. M. Will, Phys. Rev. D 88, 063522 (2013).

[6] A. Pontzen and F. Governato, Nature (London) 506, 171 (2014).

[7] F. Governato, A. Zolotov, A. Pontzen, C. Christensen, S. H. Oh, A. M. Brooks, T. Quinn, S. Shen, and J. Wadsley, Mon. Not. R. Astron. Soc. 422, 1231 (2012).

[8] M. Kuhlen, J. Guedes, A. Pillepich, P. Madau, and L. Mayer, Astrophys. J. 765, 10 (2013).

[9] A. Abramowski et al., Phys. Rev. Lett. 106, 161301 (2011).

[10] L. Pieri, J. Lavalle, G. Bertone, and E. Branchini, Phys. Rev. D 83, 023518 (2011).

[11] F. Aharonian et al., Astron. Astrophys. 457, 899 (2006).

[12] D. Berge, S. Funk, and J. Hinton, Astron. Astrophys. 466, 1219 (2007).

[13] K. Kosack et al., Astrophys. J. 608, L97 (2004).

[14] F. Aharonian et al., Astron. Astrophys. 425, L13 (2004).

[15] L. Moneta et al., Proc. Sci., ACAT2010 (2010) 57.

[16] H. Dickinson and J. Conrad, Astropart. Phys. 41, 17 (2013).

[17] T-P. Li and Y-Q. Ma, Astrophys. J. 272, 317 (1983).

[18] M. Doro et al., Astropart. Phys. 43, 189 (2013).

[19] A. Abramowski et al., Astropart. Phys. 34, 608 (2011). 